\title{
Burst-mode manipulation of magnonic vortex crystals
}

\author{
Max Hänze, ${ }^{1, *}$ Christian F. Adolff, ${ }^{1}$ Markus Weigand, ${ }^{2}$ and Guido Meier ${ }^{3,4}$ \\ ${ }^{1}$ Institut für Angewandte Physik und Zentrum für Mikrostrukturforschung, Universität Hamburg, 20355 Hamburg, Germany \\ ${ }^{2}$ Max-Planck Institute for Intelligent Systems, Heisenbergstrasse 3, 70569 Stuttgart, Germany \\ ${ }^{3}$ The Hamburg Centre for Ultrafast Imaging, Luruper Chaussee 149, 22761 Hamburg, Germany \\ ${ }^{4}$ Max-Planck Institute for the Structure and Dynamics of Matter, Luruper Chaussee 149, 22761 Hamburg, Germany
}

(Received 11 November 2014; revised manuscript received 29 January 2015; published 27 March 2015)

\begin{abstract}
The manipulation of polarization states in $4 \times 4$ vortex crystals using sinusoidal magnetic field bursts is investigated by means of a broadband ferromagnetic-resonance setup. Magnetic field excitation with the proper amplitude and frequency allows tuning different polarization states, which are observed in the measured absorption spectra. The variation of the sinusoidal burst width consecutively identifies the time scale of the underlying process. A memorylike polarization state writing process is demonstrated on the submicrosecond time scale.
\end{abstract}

DOI: 10.1103/PhysRevB.91.104428

PACS number(s): 75.70.Kw, 61.72.-y, 68.37.Yz, 76.50.+g

\section{INTRODUCTION}

Vortices in magnetic micro- and nanostructures [1] are in the focus of basic research as well as of applied sciences because their unique properties can be tailored to fulfill broad requirements, from memory applications [2] to targeted cancer-cell destruction [3]. Such structures can be spatially arranged so that the interaction between the elements leads to new remarkable properties of the whole ensemble [4,5]. So-called magnonic crystals are comparable to photonic crystals. Both feature a density of states and a band structure. We investigate interacting magnetic vortices as building blocks of a magnonic vortex crystal. Vortices form in magnetic nanodisks of suitable geometry [1]. They have a magnetization configuration curling in the plane either clockwise or counterclockwise, with an out-of-plane component at the center position pointing either up or down. This entails two state parameters, chirality $C$ and polarization $p$ [6]. The gyrotropic mode representing the fundamental excitation of the vortex ground state can be compared to the oscillation of a harmonic oscillator [7]. Here, the vortex cores gyrate around the equilibrium position of the disk. Coupling occurs when the center-to-center distance of a pair of vortices is less than twice the diameter of the disks [8,9]. The interaction between the elements has been studied for pairs [10-12], chains [13], and two-dimensional arrangements [14-16] of vortices. In contrast to systems of coupled harmonic oscillators, the interaction strength can be tuned dynamically, in dependence on the polarizations [11,1719]. Controlling the vortex polarizations allows tuning the properties of a magnonic vortex crystal. Recently, it has been demonstrated that polarization states can be tuned using an adiabatically decreasing harmonic excitation [16,20]. This mechanism can also be used for $4 \times 4$ crystals, as depicted in Fig. 1(a). Depending on the frequency of an adiabatically decreasing harmonic excitation, different polarization patterns are tuned. The two depicted frequencies yield alternating and uniform vortex-core polarizations for 210 and $260 \mathrm{MHz}$, respectively. The polarizations of the vortices within the array are obtained from the magnetic out-of-plane contrast in the center region of the disks. They have been imaged

\footnotetext{
*mhaenze@physnet.uni-hamburg.de
}

using time-resolved scanning transmission X-ray microscopy at the MAXYMUS beamline of the synchrotron BESSY II in Berlin, Germany. Different polarization states lead to different resonance frequencies of the excited eigenmodes in the crystal [21]. These polarization states can be identified using ferromagnetic-resonance (FMR) measurements.

Here, we present a method where a continuous radiofrequent excitation with a defined duration is used to tune polarization states. The polarization states [Fig. 1(a)] are identified by absorption spectra obtained from FMR measurements. This method gives insight into the time dependence of the state-formation process and yields a drastic increase in the number of state-formation cycles for a given time period. In a first step we observe the formation of polarization states for a proper choice of the frequency and the amplitude of the applied magnetic field burst [Fig. 1(b)]. In a second step the burst length is varied to identify the time scale of the state-formation process. It is shown that states can be tuned within a small number of periods of the applied harmonic signal. The presented method is then used to achieve a memorylike writing process between polarization states on the submicrosecond time scale.

\section{SAMPLE FABRICATION \& METHODS}

Arrays of polycrystalline permalloy $\left(\mathrm{Ni}_{80} \mathrm{Fe}_{20}\right)$ disks depicted in Fig. 1(c) are prepared by electron-beam lithography, thermal evaporation, and lift-off processing on a silicon substrate with a $300 \mathrm{~nm}$ silicon-oxide coating. X-ray microscopy has been performed on samples prepared on silicon-nitride membranes with a thickness of $100 \mathrm{~nm}$. As shown in Fig. 1(d), the disks have a diameter $d$ of $2 \mu \mathrm{m}$, a thickness $t$ of $60 \mathrm{~nm}$, and a center-to-center distance $D$ of $2.25 \mu \mathrm{m}$. A coplanar waveguide is deposited on top of the arrays via thermal evaporation of $120 \mathrm{~nm}$ of copper and a protection layer of $5 \mathrm{~nm}$ of gold. The waveguide covers 30 vortex arrays that are addressed as an ensemble of vortex crystals in the following. A sinusoidal signal is driven through the signal line of the coplanar waveguide, leading to an alternating magnetic field acting in the plane of the ferromagnetic elements. Measurements are performed using a broadband FMR setup with a high-frequency signal generator and an averaging power meter. Sinusoidal field bursts depicted in Fig. 1(b) are 
(a)



(b)
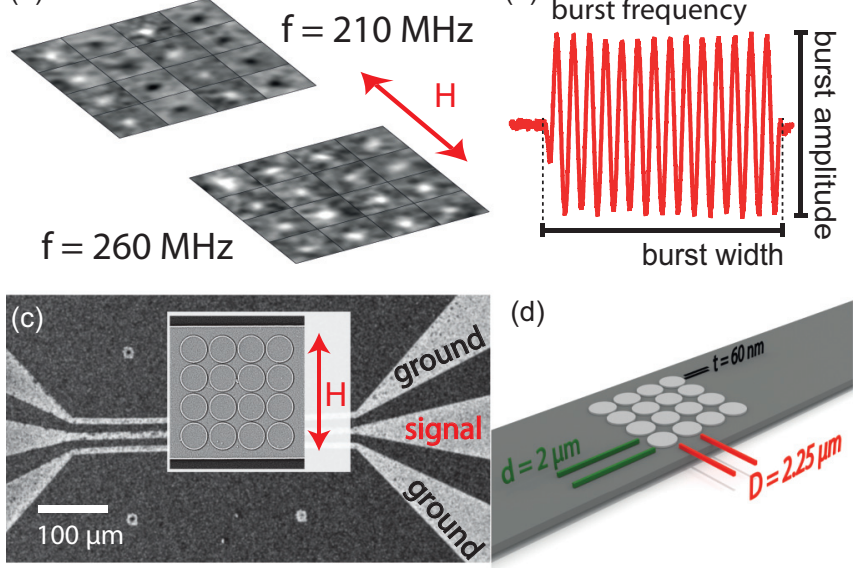

FIG. 1. (Color online) (a) Polarization patterns of the $4 \times 4$ vortex crystal obtained from scanning transmission $x$-ray microscopy for two different state-formation frequencies. The region of the magnetic vortex core is shown. The polarizations are depicted by black $(p=-1)$ and white $(p=1)$ contrast. (b) Sinusoidal magnetic field burst with defined burst width, amplitude, and frequency ( $80 \mathrm{~ns}$, $1.3 \mathrm{mT}$, and $190 \mathrm{MHz}$ in the example). (c) Ground-signal-ground geometry of the waveguide on top of the arrays of ferromagnetic disks. The inset shows a $4 \times 4$ array of the disks. (d) Scheme of the investigated $4 \times 4$ vortex crystal.

applied by the integrated pulse modulation option of the signal generator prior to the FMR absorption measurements. The field bursts are adjusted to distinct burst widths, frequencies, and amplitudes.

\section{EXPERIMENTS}

Figure 2 shows FMR absorption spectra after a sinusoidal magnetic field burst with frequencies of $190 \mathrm{MHz}$ [Fig. 2(a)] and $260 \mathrm{MHz}$ [Fig. 2(b)] and a width of $5 \mu \mathrm{s}$. The burst amplitude is varied from 100 to $2900 \mathrm{~A} / \mathrm{m}$. For low and high amplitudes the measured absorption spectra are identical. They correspond to the absorption of random polarization states within the ensemble of $4 \times 4$ vortex crystals. At intermediate burst amplitudes the absorption peak shifts away from the frequency of the field burst. This shift of the resonance frequency is attributed to the formation of polarization states. The maximum amplitude, $550 \mathrm{~A} / \mathrm{m}$ [Fig. 2(a)] or $1300 \mathrm{~A} / \mathrm{m}$ [Fig. 2(b)], leading to state formation depends on the burst frequency. The x-ray measurements have been performed for state-formation frequencies of 210 and $260 \mathrm{MHz}$. According to these measurements a frequency of $210 \mathrm{MHz}$ predominantly generates the stripe pattern presented in Fig. 1(a). This pattern is also generated at $190 \mathrm{MHz}$, according to the calculations that are presented in the following. For a frequency of $260 \mathrm{MHz}$ the pattern with uniform polarizations can be generated. To analytically describe the experimental data, we calculate the field-driven vortex-core motions for every possible polarization pattern of the vortex array. Therefore, we use a theoretical model where the coupling between the vortices is derived by surface charges that emerge when a vortex is deflected by a vector $\vec{X}$ from its equilibrium position [22]. The model is based on the analytical Thiele model [23]. The
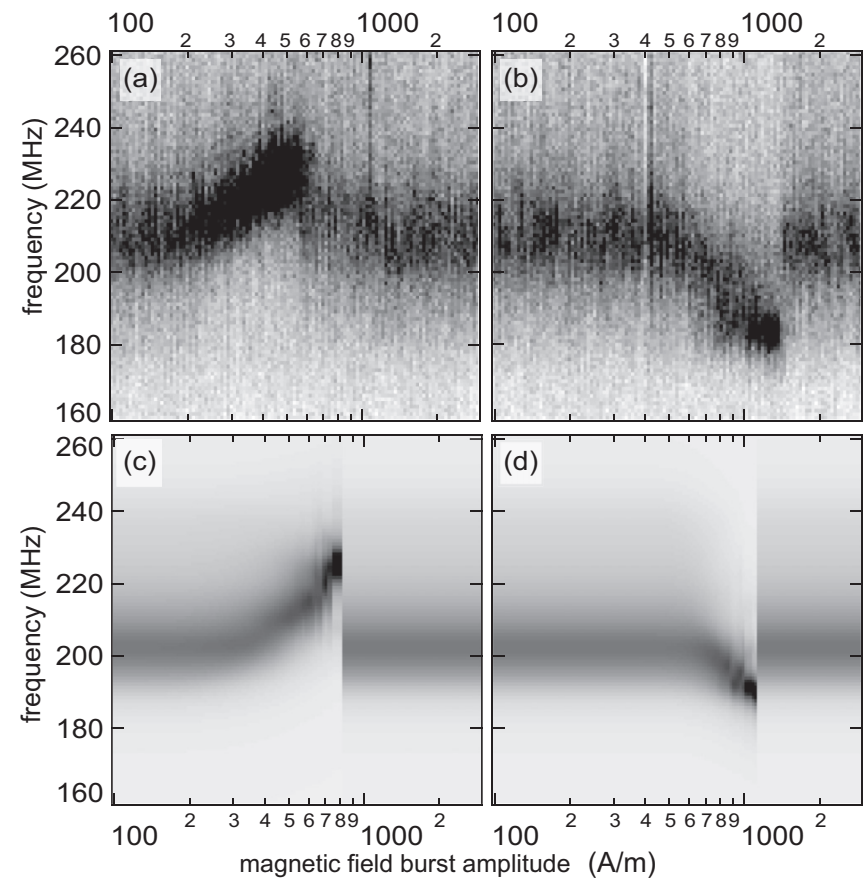

FIG. 2. (a), (b) Absorption spectra of the ensemble of arrays of $4 \times 4$ magnetic vortices after the application of a sinusoidal magnetic field burst with a width of $5 \mu \mathrm{s}$. The burst amplitude is varied. The frequency of the applied field is (a) $190 \mathrm{MHz}$ and (b) $260 \mathrm{MHz}$. Calculated absorption spectra for the two frequencies (c) $190 \mathrm{MHz}$ and (d) $260 \mathrm{MHz}$. The spectra are calculated from the ensemble of all stable states for different burst amplitudes.

minimization of all micromagnetic energies is expressed by a force $\vec{F}=-\vec{\nabla} E$. The exchange and demagnetization energy is described by a two-dimensional harmonic potential $E_{\text {harm }}$ [24]. A Zeeman term $E_{\text {zee }}$ takes external magnetic fields into account [24]. The interaction between neighboring vortices $j$ and $k$ is described via the magnetostatic energy between the side surfaces that reads $E_{\text {int }}=\xi C_{j} C_{k}\left(\hat{\eta}_{j k} \cdot \vec{X}_{k}\right) \cdot \vec{X}_{j}$, with a scaling factor $\xi$ and the interaction matrix $\hat{\eta}$ depending on the geometry, the magnetic properties, and the relative position of the coupled vortices [18]. The scaling factor $\xi=0.7$ takes into account the theoretically predicted and experimentally observed fact that the coupling is overestimated by the rigidvortex model $[10,25]$. The calculations are performed using the same spatial dimensions of the crystal as described for the experimental structures. A saturation magnetization $M_{S}$ of permalloy of $800 \mathrm{kA} / \mathrm{m}$ and a resonance frequency of an isolated vortex of $\omega_{0} /(2 \pi)=220 \mathrm{MHz}$ with a damping coefficient [26] $\Gamma=2 \omega_{0} \alpha$ is assumed. A Gilbert damping of $\alpha=0.01$ has been used in the calculations. The steady-state motions of the vortices are calculated numerically for each of the $2^{16}=65536$ polarization patterns at different frequencies. The absorption spectrum $A_{i}(f, H)$ corresponding to the sum of the squared velocities of the vortex crystal [27] at different frequencies $f$ and field amplitudes $H$ is derived for each polarization pattern $i$. The averaged absorption spectrum $A_{\text {avg }}$ depends on the predominant polarization patterns. For the 
statistical ensemble it is given by

$$
A_{\mathrm{avg}}\left(f, f_{s}, H\right)=\frac{1}{Z_{s}} \sum_{i=1}^{2^{16}} P_{i}\left(f_{s}, H\right) A_{i}\left(f, H_{0}\right),
$$

where $Z_{s}=\sum_{j=1}^{2^{16}} P_{j}\left(f_{s}, H\right)$ is the number of stable states, $f_{s}$ is the state-formation frequency, $H$ is the amplitude of the field burst, and $H_{0}$ is the constant measurement amplitude of $44 \mathrm{~A} / \mathrm{m}$. The given measurement amplitude warrants a noninvasive detection of the absorption spectra. The probability that a state is stable is given by

$$
P_{i}\left(f_{s}, H\right)=\Theta\left(A_{s}-A_{i}\left(f_{s}, H\right)\right),
$$

according to the stability criterion presented in Ref. [16]. The Heaviside theta function is equal to 1 when the mean of the squared velocities is below a certain threshold $A_{s}$. In this case the state is stable. We find that a value of $A_{s}=(250 \mathrm{~m} / \mathrm{s})^{2}$ fits the experiments. It is in agreement with previous work on vortex-core switching [26]. The polarization switches when the velocities of the vortices reach the critical velocity of $250 \mathrm{~m} / \mathrm{s}$. When the burst amplitude is high enough, all polarization patterns are unstable. In this case the number of stable states $Z_{s}$ is zero. The system does not settle in a certain polarization pattern. It rather switches continuously between the states. The averaged absorption is identical to the case when all patterns are stable. The absorption spectra depicted in Figs. 2(c) and 2(d) are calculated for different field amplitudes and the two different state-formation frequencies, 190 and $260 \mathrm{MHz}$. Due to small variations in the prepared structures and differences in the $4 \times 4$ crystals of the ensemble, the experimental measurements smear out. We observe a slight mismatch regarding the shift in the magnetic field burst amplitude between experiment and calculation. This shift cannot be understood by our model at first glance. However, the vortex dynamics for large trajectories tends towards an anharmonic regime [27] and thereby to a slight change in the resonance frequency. This effect has not been regarded by our model, and may explain the addressed differences. Nevertheless, the theoretical prediction is in good agreement with the experimental results for both frequencies. Compared to a $3 \times 3$ crystal that has nine vortices investigated in Ref. [16], the number of possible polarization patterns (65 536) is 128 times larger for the $4 \times 4$ vortex crystal. Thus, the number of possible excitable eigenmodes is drastically increased. This results in a continuous spectrum of resonances as observed in Fig. 2.

Using the Thiele approach we can determine the absorption spectra of the ensemble of vortex crystals. However, the dynamics of the switching process is not accessible by this model. To understand the time dependence of the state formation, we performed additional measurements where the duration of the sinusoidal field burst is varied. Figure 3 depicts absorption spectra for two different burst frequencies, $190 \mathrm{MHz}$ [Fig. 3(a)] and $260 \mathrm{MHz}$ [Fig. 3(b)], in dependence on the sinusoidal burst duration. The field amplitude has been adjusted so that only very few states are stable for both frequencies, respectively. We observe that for a burst width above 100 ns the absorption spectra remain on the same level, i.e., the polarization state is reliably tuned. The state formation

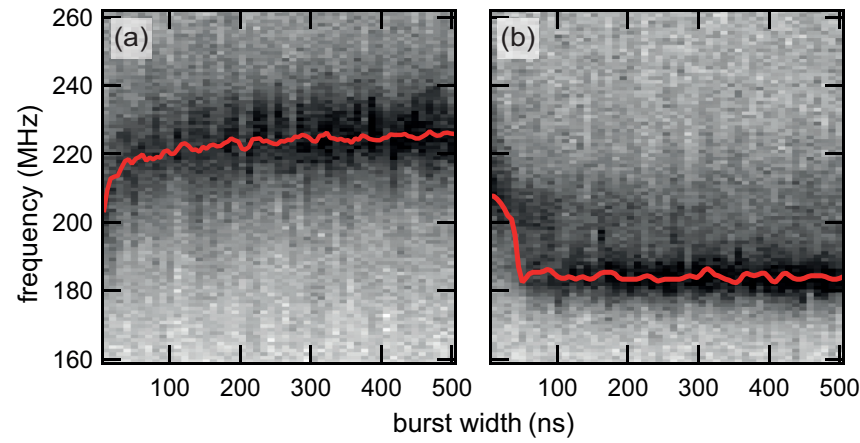

FIG. 3. (Color online) Absorption spectra of the ensemble of arrays of $4 \times 4$ magnetic vortices for a constant burst amplitude of (a) $500 \mathrm{~A} / \mathrm{m}$ and (b) $1200 \mathrm{~A} / \mathrm{m}$, in dependence on the burst width. The frequency of the sinusoidal field burst is (a) $190 \mathrm{MHz}$ and (b) $260 \mathrm{MHz}$. The red line indicates a smoothed fit to the maximum of the absorption to guide the eye. The time scale of the polarization state formation is about $100 \mathrm{~ns}$.

can be interpreted as a path through a potential landscape of vortex patterns. The oscillating magnetic field excites the vortices until they switch from an unstable into a random state. This process ceases when a certain stable state is reached. The time scale to reach a stable state can be identified to be about $100 \mathrm{~ns}$ for the present material and geometry. This knowledge about the time scale of the state-formation process enables us to demonstrate a writing process on the submicrosecond time scale.

Figure 4 depicts the absorption spectra of the ensemble of vortex crystals along the consecutive application of different magnetic field bursts. The peaks of the absorption spectra change depending on the characteristics of the two write bursts. We use burst frequencies of $f_{1}=180 \mathrm{MHz}$ and $f_{2}=260 \mathrm{MHz}$ with burst amplitudes of 500 and $1250 \mathrm{~A} / \mathrm{m}$, respectively. The write bursts have a length of 200 ns. In Fig. 4(b) the two different writing intervals are indicated by light blue and red regions. We observe that the polarization states are tuned independently of the previous polarization state.

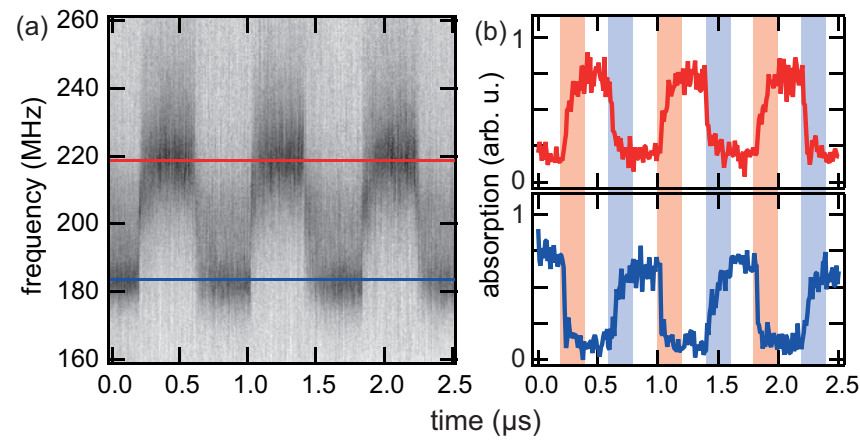

FIG. 4. (Color online) (a) Absorption spectra of the writing process between two polarization states over time. (b) Absorptions at the indicated frequencies of the absorption spectra (red and blue). The writing bursts are indicated by light blue and light red regions. They have frequencies of (1) $180 \mathrm{MHz}$ and (2) $260 \mathrm{MHz}$ and a length of $200 \mathrm{~ns}$. The burst amplitude is (1) $500 \mathrm{~A} / \mathrm{m}$ and (2) $1250 \mathrm{~A} / \mathrm{m}$. The writing process is independent of the prior polarization state. 
Each absorption pattern bears certain information. Thus, by using different write burst characteristics, different absorption spectra can be generated. This allows one to store and read the polarization states in a vortex crystal by the application of high-frequency currents through a single stripline.

\section{CONCLUSION}

We presented a method to reliably tune polarization states of magnonic vortex crystals on the submicrosecond time scale. The absorption spectra of the measured states can be theoretically predicted using a rigid particle model. This way the measured absorption spectra are understood by the average of a statistical ensemble of stable polarization states. Our work allows further research studies towards potential storage devices using magnetic vortex crystals. The formation of polarization states allows tailoring characteristic properties of magnonic vortex crystals, such as the band structure or the density of states.

\section{ACKNOWLEDGMENTS}

We thank Ulrich Merkt and Andreas Vogel for fruitful discussions and Michael Volkmann for superb technical assistance. We acknowledge the support of the Max-PlanckInstitute for Intelligent Systems (formerly MPI for Metals Research), Department Schütz and the MAXYMUS team, particularly Michael Bechtel and Eberhard Goering. Financial support of the Deutsche Forschungsgemeinschaft via the Sonderforschungsbereich 668 and the Graduiertenkolleg 1286 is gratefully acknowledged. This work has been supported by the excellence cluster "The Hamburg Centre for Ultrafast Imaging (CUI): Structure, Dynamics and Control of Matter at the Atomic Scale" of the Deutsche Forschungsgemeinschaft.
[1] T. Shinjo, T. Okuno, R. Hassdorf, K. Shigeto, and T. Ono, Science 289, 930 (2000).

[2] S. Bohlens, B. Krüger, A. Drews, M. Bolte, G. Meier, and D. Pfannkuche, Appl. Phys. Lett. 93, 142508 (2008).

[3] D.-H. Kim, E. A. Rozhkova, I. V. Ulasov, S. D. Bader, T. Rajh, M. S. Lesniak, and V. Novosad, Nat. Mater. 9, 165 (2010).

[4] V. V. Kruglyak, S. O. Demokritov, and D. Grundler, J. Phys. D: Appl. Phys. 43, 264001 (2010).

[5] B. Lenk, H. Ulrichs, F. Garbs, and M. Münzenberg, Phys. Rep. 507, 107 (2011).

[6] A. Wachowiak, J. Wiebe, M. Bode, O. Pietzsch, M. Morgenstern, and R. Wiesendanger, Science 298, 577 (2002).

[7] B. Van Waeyenberge, A. Puzic, H. Stoll, K. W. Chou, T. Tyliszczak, R. Hertel, M. Fähnle, H. Brück, K. Rott, G. Reiss, I. Neudecker, D. Weiss, C. H. Back, and G. Schütz, Nature (London) 444, 461 (2006).

[8] A. Vogel, A. Drews, T. Kamionka, M. Bolte, and G. Meier, Phys. Rev. Lett. 105, 037201 (2010).

[9] J. Mejía-López, D. Altbir, A. H. Romero, X. Batlle, I. V. Roshchin, C.-P. Li, and I. K. Schuller, J. Appl. Phys. 100, 104319 (2006).

[10] S. Sugimoto, Y. Fukuma, S. Kasai, T. Kimura, A. Barman, and Y. C. Otani, Phys. Rev. Lett. 106, 197203 (2011).

[11] A. Vogel, T. Kamionka, M. Martens, A. Drews, K. W. Chou, T. Tyliszczak, H. Stoll, B. Van Waeyenberge, and G. Meier, Phys. Rev. Lett. 106, 137201 (2011).

[12] H. Jung, K.-S. Lee, D.-E. Jeong, Y.-S. Choi, Y.-S. Yu, D.-S. Han, A. Vogel, L. Bocklage, G. Meier, M.-Y. Im, P. Fischer, and S.-K. Kim, Sci. Rep. 1, 59 (2011).
[13] D.-S. Han, A. Vogel, H. Jung, K.-S. Lee, M. Weigand, H. Stoll, G. Schütz, P. Fischer, G. Meier, and S.-K. Kim, Sci. Rep. 3, 2262 (2013).

[14] J. Shibata and Y. Otani, Phys. Rev. B 70, 012404 (2004).

[15] A. Vogel, M. Hänze, A. Drews, and G. Meier, Phys. Rev. B 89, 104403 (2014).

[16] C. F. Adolff, M. Hänze, A. Vogel, M. Weigand, M. Martens, and G. Meier, Phys. Rev. B 88, 224425 (2013).

[17] Y. Liu, Z. Hou, S. Gliga, and R. Hertel, Phys. Rev. B 79, 104435 (2009).

[18] J. Shibata, K. Shigeto, and Y. Otani, Phys. Rev. B 67, 224404 (2003).

[19] A. Barman, S. Barman, T. Kimura, Y. Fukuma, and Y. Otani, J. Phys. D: Appl. Phys. 43, 422001 (2010).

[20] S. Jain, V. Novosad, F. Y. Fradin, J. E. Pearson, V. Tiberkevich, A. N. Slavin, and S. D. Bader, Nat. Commun. 3, 1330 (2012).

[21] M. Hänze, C. F. Adolff, M. Weigand, and G. Meier, Appl. Phys. Lett. 104, 182405 (2014).

[22] K. Yu. Guslienko, B. A. Ivanov, V. Novosad, Y. Otani, H. Shima, and K. Fukamichi, J. Appl. Phys. 91, 8037 (2002).

[23] A. A. Thiele, Phys. Rev. Lett. 30, 230 (1973).

[24] B. Krüger, A. Drews, M. Bolte, U. Merkt, D. Pfannkuche, and G. Meier, Phys. Rev. B 76, 224426 (2007).

[25] O. V. Sukhostavets, J. González, and K. Y. Guslienko, Phys. Rev. B 87, 094402 (2013).

[26] M. Martens, T. Kamionka, M. Weigand, H. Stoll, T. Tyliszczak, and G. Meier, Phys. Rev. B 87, 054426 (2013).

[27] A. Drews, B. Krüger, G. Selke, T. Kamionka, A. Vogel, M. Martens, U. Merkt, D. Möller, and G. Meier, Phys. Rev. B 85, 144417 (2012). 